\title{
ON CORNER AVOIDANCE PROPERTIES OF RANDOM-START HALTON SEQUENCES
}

\author{
JÜRGEN HARTINGER ${ }^{+}$AND VOLKER ZIEGLER*
}

\begin{abstract}
Recently, the analysis of quasi-Monte Carlo (QMC) sampling of integrands with singularities gained considerable interest. In this setting error bounds for QMC integration, in addition to discrepancy, include a measure how well the singularities are avoided by the utilized sequences. The article aims to generalize results for the corner avoidance of the classical Halton sequence to Halton sequences that start in an arbritrary point of the unit cube. In particular, it is shown that almost all (in Lebesgue sense) random-start Halton sequences exhibit the same corner avoidance property as the original Halton sequence.
\end{abstract}

\section{INTRODUCTION}

Quasi-Monte Carlo methods are deterministic alternatives to classical Monte Carlo methods with asymptotically superior error bounds for integration problems when the integrand belongs to a suitable class of functions.

Let $\bar{U}^{s}=[0,1]^{s}$, the $s$-dimensional unit cube, and consider functions $f: \bar{U}^{s} \rightarrow \mathbb{R}$ such that $I=\int_{\bar{U}^{s}} f(\mathbf{x}) d \mathbf{x}$ exists. Let furthermore $\left(\mathbf{x}_{n}\right)_{n>0}$ be a sequence with $\mathbf{x}_{n}=\left(x_{n}^{(1)}, \ldots, x_{n}^{(s)}\right) \in[0,1)^{s}$. For a subset $B \subseteq \bar{U}^{s}$, denote by $\lambda(B)$ its Lebesgue measure and by $\chi_{B}$ its characteristic function, i.e. $\chi_{B}(\mathbf{x})$ equals 1 for $\mathbf{x} \in B$, and 0 otherwise. The star discrepancy of the set $\left(\mathbf{x}_{n}\right)_{1 \leq n \leq N}$, measuring its uniformness, is given by

$$
D_{N}^{*}\left(\mathbf{x}_{1}, \ldots, \mathbf{x}_{N}\right)=\sup _{J \in \mathcal{J}^{*}}\left|\frac{1}{N} \sum_{n=1}^{N} \chi_{J}\left(\mathbf{x}_{n}\right)-\lambda(J)\right|,
$$

where $\mathcal{J}^{*}$ is the set of all subintervals of $\bar{U}^{s}$ of the form $\prod_{i=1}^{s}\left[0, u_{i}\right)$. Sequences with best known order of discrepancy $\left(\mathcal{O}\left(N^{-1}(\log N)^{s}\right)\right)$ are called low-discrepancy sequences (LDS). Different LDS constructions were proposed by Halton [3], Sobol' [15], Faure [2] and Niederreiter [7, 8]. Tezuka and Tokuyama [17] show that the last three approaches can be unified by a generalization of Niederreiter's principles.

We define the QMC estimator of $I$ as $\hat{I}_{N}=\frac{1}{N} \sum_{n=1}^{N} f\left(\mathbf{x}_{n}\right)$. Hlawka's theorem [6] bounds the QMC integration error as follows:

$$
\left|I-\hat{I}_{N}\right| \leq V_{H K}(f) D_{N}^{*}\left(\mathbf{x}_{1}, \ldots, \mathbf{x}_{N}\right)
$$

where $V_{H K}(f)$ denotes the variation in the sense of Hardy and Krause (for a definition and a survey on concepts of multidimensional variation see [11]). A thorough introduction to the field of QMC integration may be found in the monograph [9].

2000 Mathematics Subject Classification. Primary 11K45; Secondary 65C05, 11J25, 11 D61.

Key words and phrases. QMC integration for singular integrands, random start Halton sequence, corner avoidance.

+ The first author was supported by the Austrian Science Foundation, project S-8308-MAT.

* The second author was supported by the Austrian Science Foundation, project S-8307-MAT. 
Mainly inspired by financial applications, the theory of QMC quadrature formulae for singular integrands (those do not have bounded Hardy-Krause variation) was intensively studied recently. In a setting where the singularities lie in the boundary, Owen [12] shows that the QMC convergence order under suitable growth conditions on the integrand in addition to the discrepancy depends on the speed with which the utilized sequence approaches the boundary of the unit cube.

The quantity of main interest within this setting is the hyperbolic distance from a point $\mathbf{z}=$ $\left(z_{1}, \ldots, z_{s}\right) \in[0,1)^{s}$ to a corner of the unit cube $\mathbf{h}=\left(h_{1}, \ldots, h_{s}\right) \in\{0,1\}^{s}$, which is defined by $\|\mathbf{z}\|_{\mathbf{h}}=\prod_{i=1}^{s}\left|z_{i}-h_{i}\right|$. In order to get (asymptotically) efficient QMC rules for these integrands one has to find point sequences satisfying the condition

$$
\exists n_{0} \in \mathbb{Z}^{+}: \forall n \geq n_{0}:\left\|\mathbf{x}_{n}\right\|_{\mathbf{h}} \geq c n^{-r},
$$

with a constant $c=c(r)>0$ and small $r$. A sequence is said to avoid the corner $\mathbf{h}$ if (1) is fulfilled with $r \leq 1+\varepsilon$ for all $\varepsilon>0$.

Let us state Owen's result [12], in order to shed some light on the role of $r$. Let $f:(0,1]^{s} \rightarrow$ $\mathbb{R}$ be a real-valued Lebesgue measurable function which is singular in the origin. Moreover, we assume some growth conditions. For a set $u \subset\{1, \ldots, s\}$ of indices, the symbol $\partial^{u} f(\mathbf{x})$ represents $\left(\prod_{j \in u} \partial / \partial x_{j}\right) f(\mathbf{x})$, with the convention that $\partial^{\emptyset} f(\mathbf{x})=f(\mathbf{x})$. Our growth condition is that

$$
\left|\partial^{u} f(\mathbf{x})\right| \leq B \prod_{j=1}^{s}\left(x_{j}\right)^{-A_{j}-\mathbf{1}_{j \in u}}
$$

holds for some $A_{j}>0$, some $B<\infty$ and all $u \subseteq\{1, \ldots, s\}$, where $\mathbf{1}_{j \in u}=1$ if $j \in u$ and $\mathbf{1}_{j \in u}=0$ otherwise. Now we can state Owen's result [12, Theorem 5.5]:

Theorem 1. Let $f(\mathbf{x})$ be as above and suppose $\mathbf{x}_{1}, \ldots, \mathbf{x}_{N}$ satisfy (1). Then for any $\eta>0$,

$$
\left|\hat{I}_{N}-I\right| \leq C_{1} D_{N}^{*}\left(\mathbf{x}_{1}, \ldots \mathbf{x}_{N}\right) N^{\eta+r \max _{j} A_{j}}+C_{2} N^{r\left(\max _{j} A_{j}-1\right)},
$$

holds for finite $C_{1}$ and $C_{2}$, that may depend on $\eta$.

A similar result also holds for other corners than $\mathbf{0 .}$

As Owen [12] remarks, the quasi-Monte Carlo integration is superior to Monte Carlo integration, if $\max _{j} A_{j}<1 /(2 r)$, provided $D_{N}^{*}\left(\mathbf{x}_{1}, \ldots \mathbf{x}_{N}\right)=O\left(N^{-1+\epsilon}\right)$. In view of applications of quasi-Monte Carlo integration of singular functions, we have to use low discrepancy sequences which avoid corners with small $r$.

In the case $\mathbf{h}=0$ the lower bound $r \geq 1$ is obvious for $(t, s$ )-sequences (for a definition see e.g. [9]) and for Halton sequences. Sobol' [16] shows that $r=1$ holds for Sobol' sequences, Owen [12] establishes $r=1$ for Halton sequences. Hartinger et al. [4] show $r=1$ for generalized Niederreiter sequences. For all $\mathbf{h}$, i.e. $\min _{\mathbf{h} \in\{0,1\}^{s}}\left\|x_{N}\right\|_{\mathbf{h}}$, much less is known: Hartinger et al. [4] establish $r \leq 1+\varepsilon$ for Halton sequences and $r \geq 3 / 2$ for the Faure sequence. In a randomized setting Owen [10] shows that the expected error of randomized QMC (under mild conditions on the moments of the integrand) is superior to Monte Carlo even if there exist point singularities with unknown locations. In this paper we show that $r \leq 1+\epsilon$ holds also for almost all random start Halton sequences.

Section 2 reviews the definition of random start Halton sequences, that might be considered as Halton sequences started at an arbitrary point in the unit cube (the classical Halton sequence starts in 0). In view of an application of Schmidt's Subspace theorem we formulate and discuss this theorem in Section 3. The Subspace theorem will be essential part in the proof of the results in section 4, 
which establishes corner avoidance results for Halton sequences started in special points of the unit cube. In section 5 we show that the set of start points inducing Halton sequences that do not avoid all corners, has Lebesgue measure zero.

\section{Random Start Halton Sequences}

For a base $p \in \mathbb{Z}^{+}$( $\mathbb{Z}^{+}$denotes the set of positive integers) and an integer $n$ with $p$-adic expansion $n=\sum_{r=1}^{l} a_{r}(n) p^{r-1}$, define the radical inverse function by $\Phi_{p}(n)=\sum_{r=1}^{l} a_{r}(n) p^{-r}$. The $n$-th element of the $s$-dimensional Halton sequence [3] in relatively coprime bases $p_{1}, \ldots, p_{s}$ (typically the first $s$ primes) is given by $\mathbf{x}_{n}=\left(\Phi_{p_{1}}(n), \ldots, \Phi_{p_{s}}(n)\right)$.

Wang and Hickernell [18] propose to generalize the Halton sequence in the following way: Let $z \in[0,1)$ be written as

$$
z=\sum_{r=1}^{\infty} \frac{a_{r}(z)}{p^{r}}
$$

where for all $r_{0} \in \mathbb{Z}^{+}$there exists an $r \geq r_{0}$ such that $a_{r}(z)<p-1$ and $1=\sum_{r=1}^{\infty} \frac{p-1}{p^{r}}$. The $p$-adic von Neumann-Kakutani transform is given by rightward-carry addition in base $p$, i.e. $T_{p}(z)=z \oplus \frac{1}{p}=$ $\frac{1+a_{m}(z)}{p^{m}}+\sum_{r>m} \frac{a_{r}(z)}{p^{r}}$, where $m=\min \left\{r \in \mathbb{Z}^{+} \mid u_{r} \neq p-1\right\}$. For relatively coprime bases $p_{1}, \ldots, p_{s}$, the $s$-dimensional $\mathbf{z}$-start Halton sequence is recursively defined by $\mathbf{z}=\mathbf{x}_{0}=\left(x_{0}^{(1)}, \ldots, x_{0}^{(s)}\right) \in[0,1]^{s}$ and $\mathbf{x}_{n+1}=\left(T_{p_{1}}\left(x_{n}^{(1)}\right), \ldots, T_{p_{s}}\left(x_{n}^{(s)}\right)\right)$ for $n \in \mathbb{Z}_{0}^{+}$. Wang and Hickernell [18] show that for all $\mathbf{z} \in[0,1]^{s}$ the induced Halton sequence is a low discrepancy sequence and propose a randomization of the Halton sequence by choosing $\mathbf{z}$ through a realization of a uniformly distributed random variable on $[0,1]^{s}$ (therefore this construction is known as random start Halton sequence).

For later convenience, we give an equivalent $p$-adic definition of the $\mathbf{z}$-start Halton sequence: For a prime $p$, let $\mathbb{Z}_{p}$ denote the set of all $p$-adic integers. Suppose $z=\sum_{r=1}^{\infty} a_{r}(z) p^{r-1} \in \mathbb{Z}_{p}$, we define the extended radical inverse function $\Phi_{p}: \mathbb{Z}_{p} \rightarrow[0,1]$, with $z \mapsto \sum_{r=1}^{\infty} a_{r}(z) p^{-r}$. Since $a_{r}(z) \in$ $\{0,1, \ldots, p-1\}$, the sum converges and $\Phi_{p}$ is a well-defined function. The $z$-start van der Corput sequence $\left(\phi_{p}(n ; z)\right)_{n \geq 1}$ for a starting point $z \in \mathbb{Z}_{p}$ is defined by $\phi_{p}(n ; z)=\Phi_{p}(n+z)$. Finally, for distinct primes $p_{1}, \ldots, p_{s}$ and fixed $p_{i}$-adic integers $z_{i},(1 \leq i \leq s)$, the $\mathbf{z}$-start Halton sequence $\left(\mathbf{x}_{n}\right)_{n \geq 1}$ is given by

$$
\mathbf{x}_{n}=\left(\phi_{p_{1}}\left(n ; z_{1}\right), \ldots, \phi_{p_{s}}\left(n ; z_{s}\right)\right) .
$$

For $k \in \mathbb{Z}^{+}$, the sequence $\left(\phi_{p}(n ; k)\right)_{n \geq 1}=\left(\Phi_{p}(n+k)\right)_{n \geq 1}$ is a shift of the van der Corput sequence by $k$ elements. Therefore a $\mathbf{z}$-start Halton sequence with $\mathbf{z} \in \mathbb{Z}^{s}$ is called a finitely shifted Halton sequence. If there exists a coordinate in $\mathbf{z}$ such that $z_{i} \in \mathbb{Z}_{p} \backslash \mathbb{Z}$, the corresponding Halton sequence is said to be infinitely shifted.

Let us define the map $\tilde{\Phi}_{p}(z):[0,1] \rightarrow \mathbb{Z}_{p}$ with $z \mapsto \sum_{r=1}^{\infty} a_{r}(z) p^{r-1}$, where the coefficients are obtained by (3). Thus, we have $\Phi_{p} \circ \tilde{\Phi}_{p}=$ id. For $\mathbf{z}=\left(z_{1}, \ldots, z_{s}\right) \in[0,1)^{s}$ and $n \in \mathbb{Z}^{+}$, the corresponding $\mathbf{z}$-start Halton sequence may now be written in the following form

$$
\mathbf{x}_{n}:=\mathbf{x}_{n}^{\left(z_{1}, \ldots, z_{s}\right)}=\left(\phi_{p_{1}}\left(n ; \tilde{\Phi}\left(z_{1}\right)\right), \ldots, \phi_{p_{s}}\left(n ; \tilde{\Phi}\left(z_{s}\right)\right)\right) .
$$

Remark: Note that we have defined the Halton sequence only for prime basis. A similar construction may be obtained for relative prime basis. Observe that for a prime $p, \mathbb{Z}_{p}$ may be defined by $\mathbb{Z}_{p}=\lim _{\leftarrow} \mathbb{Z} / p^{k} \mathbb{Z}$ the inverse limit of the rings $\mathbb{Z} / p^{k} \mathbb{Z}$. This inverse limit exists even if $p$ is not a prime. 
Considered as a limit of rings it does not give a suitable structure, i.e in general $\mathbb{Z}_{p}$ is not a domain. Nevertheless viewed as a limit of additive groups one gets a similar structure as in the prime case. Since in the definition above only the additive structure is needed, the definition is suitable to any relatively coprime basis.

\section{The Subspace Theorem}

Akin to the classical Halton sequence (cf. [4]) the backbone in the proof for finitely shifted Halton sequences (see Section 4) is the $p$-adic Subspace theorem due to Schlickewei [13] (see also [14, Chapter $\mathrm{V}$, Theorem 1D]). In the literature there exist many versions of the Subspace theorem. The most general form was established by Evertse and Schlickewei [1]. Because of technical reasons we only state a non quantitative version of this theorem. This version can be found in Schmidt's book [14, Chapter V]. Before we state the Subspace theorem we introduce some notations.

Let $\mathbb{Q}$ be the field of rationals, then there exist several absolute values on $\mathbb{Q}$. One of them is the so called Archimedian absolute value $|\cdot|_{\infty}$, which is the usual absolute value. For any prime $p$ we obtain an absolute value $|\cdot|_{p}$, with $|x|_{p}=p^{-\alpha}$, where $x=p^{\alpha} u / v$ with $p \not \chi u v$, these absolute values are called non-Archimedian. The Archimedian and the non-Archimedian absolute values form the set $M(\mathbb{Q})$, the canonical absolute value. Let $K$ be some number field then every absolute value can be extended to an absolute value on $K$, possibly in several ways. Absolute values obtained in this way are called Archimedian again, if they are extensions of the usual absolute value and are called non-Archimedian if they are induced by an absolute value of the form $|\cdot|_{p}$ with $p$ a prime. The union of these absolute values is denoted by $M(K)$ and is called the set of canonical absolute values. Let $p \in\{\infty, 2,3,5 \ldots\}=M(\mathbb{Q})$ (we identify $p$ with $|\cdot|_{p}$ ) and let $\nu \in M(K)$, then we write $\nu \mid p$ if $\nu$ is induced by $p$. Again let $\nu \in M(K)$ with $\nu \mid p$. Then we denote by $K_{\nu}$ the (topological) closure of $K$ with respect to $\nu$. The index $n_{\nu}:=\left[K_{\nu}: \mathbb{Q}_{p}\right]$ is called the local degree. Note that if $p=\infty$, then $n_{\nu}=1,2$ depending on whether the $K_{\nu}$ is real or complex. We also use the following notation. Let $\mathbf{x} \in K^{s}$ then we use the notation

$$
\overline{|\mathbf{x}|}=\max _{\substack{1 \leq i \leq s \\ \nu \in M(K), \nu \mid \infty}}\left|x_{i}\right|_{\nu}
$$

Note that by linear forms we mean homogeneous polynomials of degree 1 over some number field $K$. We say that linear forms are linearly independent if they are linearly independent over their (fixed) field of coefficients. Now we can state a non-quantitative form of the Subspace theorem (see [14, Chapter V, Theorem 1D] - note the missprint non-Archimedian instead of Archimedian).

Theorem 2. Let $K$ be an algebraic number field and let $S \subset M(K)$ be a finite set of absolute values containing all of the Archimedian ones. For each $\nu \in S$ let $L_{\nu, 1}, \ldots, L_{\nu, s}$ be $s$ linearly independent (over $K$ ) linear forms in $s$ variables with coefficients in $K$. Then for given $\delta>0$, the solutions of the inequality

$$
\prod_{\nu \in S} \prod_{i=1}^{s}\left|L_{\nu, i}(\mathbf{x})\right|_{\nu}^{n_{\nu}}<\overline{\mid \mathbf{x}}^{-\delta}
$$

with $\mathbf{x} \in \mathfrak{o}_{K}^{s}$ and $\mathbf{x} \neq \mathbf{0}$, where $\mathfrak{o}_{K}$ is the ring of integers of $K$, lie in finitely many proper subspaces of $K^{s}$

In view of the next section we only need Theorem 2 in the case of $K=\mathbb{Q}$. Therefore we state also following version of the Subspace theorem.

Theorem 3. Let $\{\infty\} \subset S \subset M(\mathbb{Q})$ be a finite set of absolute values. For each $\nu \in S$ let $L_{\nu, 1}, \ldots, L_{\nu, s}$ be $s$ rational, linearly independent linear forms in $s$ variables. Then for given $\delta>0$, the solutions of 
the inequality

$$
\prod_{\nu \in S} \prod_{i=1}^{s}\left|L_{\nu, i}(\mathbf{x})\right|_{\nu}<\left(\max _{1 \leq i \leq s}\left|x_{i}\right|\right)^{-\delta}
$$

with $\mathbf{x} \in \mathbb{Z}^{s}$ and $\mathbf{x} \neq \mathbf{0}$, lie in finitely many proper subspaces of $\mathbb{Q}^{s}$.

\section{Finite Shifts}

In this section we show that almost all finite shifts preserve the corner avoidance properties of the Halton sequence in basis $p_{1}, \ldots, p_{s}$. The first step is to establish a relation between the hyperbolic distance and exact powers of basis elements dividing certain $p$-adic integers. For a $p$-adic integer $z$, we say $\alpha$ is the exact power of $p$ dividing $z$ (in symbols $p^{\alpha} \| z$ ), if $\alpha=\min \left\{r \geq 0: a_{r+1}(z) \neq 0\right\}$.

Lemma 1. Let $\mathbf{h}$ be a corner of the unit cube and let $\mathbf{z} \in[0,1]^{s}$ such that $p_{i}^{\alpha_{i}} \|\left(\tilde{\Phi}_{p_{i}}\left(z_{i}\right)+h_{i}\right)$ for $i=1, \ldots, s$. Then

$$
\prod_{i=1}^{s} p_{i}^{-\alpha_{i}-1} \leq\|\mathbf{z}\|_{\mathbf{h}} \leq \prod_{i=1}^{s} p_{i}^{-\alpha_{i}}
$$

Proof. Obviously, it suffices to prove

$$
p_{i}^{-\alpha_{i}-1} \leq\left|z_{i}-h_{i}\right| \leq p_{i}^{-\alpha_{i}}
$$

for $i=1, \ldots, s$

First, consider the case $h_{i}=0$. For notational convenience omit the index $i$. Due to $p^{\alpha} \| \tilde{\Phi}(z)$, we have $\tilde{\Phi}(z)=\sum_{r=\alpha+1}^{\infty} a_{r}(z) p^{r-1},\left(a_{\alpha+1}(z) \neq 0\right)$. Since $\Phi$ is the left inverse of $\tilde{\Phi}$, we obtain

$$
z=\Phi \circ \tilde{\Phi}(z)=\sum_{r=\alpha+1}^{\infty} \frac{a_{r}(z)}{p^{r}}, \quad a_{\alpha+1}(z) \neq 0 .
$$

Now, $p^{-\alpha-1} \leq z \leq p^{-\alpha}$ follows immediately by $0 \leq a_{r}(z) \leq p-1$ for $r \geq 1$.

Consider the case $h_{i}=1$. Omitting indices again, we have $p^{\alpha} \|(\tilde{\Phi}(z)+1)$. Hence, $\tilde{\Phi}(z)+1=$ $\sum_{r=\alpha+1}^{\infty} a_{r}(z) p^{r-1},\left(a_{\alpha+1}(z) \neq 0\right)$, and

$$
\tilde{\Phi}(z)=\sum_{r=1}^{\alpha}(p-1) p^{r-1}+\left(a_{\alpha+1}(z)-1\right) p^{\alpha}+\sum_{r=\alpha+2}^{\infty} a_{r}(z) p^{r-1} .
$$

Again, an application of $\Phi$ to $\tilde{\Phi}(z)$ yields

$$
z=\Phi \circ \tilde{\Phi}(z)=\sum_{r=1}^{\alpha} \frac{p-1}{p^{r}}+\frac{a_{\alpha+1}(z)-1}{p^{\alpha+1}}+\sum_{r=\alpha+2}^{\infty} \frac{a_{r}(z)}{p^{r}}
$$

and furthermore

$$
\begin{aligned}
1-z & =\sum_{r=1}^{\infty} \frac{p-1}{p^{r}}-\sum_{r=1}^{\alpha} \frac{p-1}{p^{r}}-\frac{a_{\alpha+1}(z)-1}{p^{\alpha+1}}-\sum_{r=\alpha+2}^{\infty} \frac{a_{r}(z)}{p^{r}} \\
& =\frac{p-a_{\alpha+1}(z)}{p^{\alpha+1}}+\sum_{r=\alpha+2}^{\infty} \frac{p-1-a_{r}(z)}{p^{r}}
\end{aligned}
$$

Thus, $p^{-\alpha-1} \leq 1-z \leq p^{-\alpha}$. 
Theorem 4. For $\mathbf{k}=\left(k_{1}, \ldots, k_{s}\right) \in \mathbb{Z}^{s}$, let

$$
\mathbf{x}_{n}=\left(x_{n}^{(1)}, \ldots, x_{n}^{(s)}\right)=\left(\phi_{p_{1}}\left(n ; k_{1}\right), \ldots, \phi_{p_{s}}\left(n ; k_{s}\right)\right)
$$

be the $\mathbf{k}$-start Halton sequence in relatively coprime basis $p_{1}, \ldots, p_{s}$. Denote by $K(L)$ the set of all shift-vectors $\mathbf{k}$ with $\|\mathbf{k}\|_{\infty}<L$ such that the $\mathbf{k}$-start Halton sequence does not avoid all corners, i.e.

$$
K(L)=\left\{\mathbf{k} \in([0, L] \cap \mathbb{Z})^{s}: \exists \varepsilon>0 \exists h \in\{0,1\}^{s} \forall n_{0} \in \mathbb{Z}^{+} \exists n \geq n_{0}:\left\|\mathbf{x}_{n}\right\|_{\mathbf{h}}<n^{-1-\epsilon}\right\} .
$$

Then

$$
\lim _{L \rightarrow \infty} \frac{\# K(L)}{L^{s}}=0
$$

Proof. Let $1 \leq i \leq s$. Let us first assume that all quantities $k_{i}+h_{i}$ are the same, say equal to $\beta$. By the virtue of Lemma 1, it suffices to show that for every $\epsilon>0$ there are only finitely many $n \in \mathbb{Z}^{+}$, such that

$$
p_{i}^{\alpha_{i}} \|\left(n+k_{i}+h_{i}\right)=n+\beta \quad \text { and } \quad \prod_{i=1}^{s} p_{i}^{\alpha_{i}} \geq n^{1+\epsilon} .
$$

Since our assumption we have $\prod_{i=1}^{s} p_{i}^{\alpha_{i}} \mid(n+\beta)$ and therefore (4) can hold only for finitely many $n$.

Now let us assume not all $k_{i}+h_{i}$ are the same. Let us write $x_{i}=n+k_{i}+h_{i}=C_{i} p_{i}^{\alpha_{i}}$. Note that $C_{i}$ and $p_{i}$ are relative prime. We may assume that the quantities $k_{i}+h_{i}$ are pairwise distinct. (If $k_{i}+h_{i}=k_{j}+h_{j}$ for two indices $i \neq j$ set $x_{i}=x_{j}=\tilde{C}_{i} p_{i}^{\alpha_{i}} p_{j}^{\alpha_{j}}$.) Fix some index $j$ and assume that $\frac{C_{1} \cdots C_{s}}{\prod_{i \neq j} x_{i}} \geq n^{-\epsilon / 2}$. For $n$ large (such that $n^{1+\epsilon / 2} \geq n+k_{j}+h_{j}=x_{j}$ ), we obtain

$$
n^{1+\epsilon} \geq x_{j} n^{\epsilon / 2} \geq x_{j} \frac{\prod_{i \neq j} x_{i}}{\prod_{i=1}^{s} C_{i}}=\prod_{i=1}^{s} p_{i}^{\alpha_{i}} .
$$

Now, assume that $\frac{C_{1} \cdots C_{s}}{\prod_{i \neq j} x_{i}} \leq n^{-\epsilon / 2}$. We come to the key point of the proof, the application of the Subspace theorem (Theorem 3). Let $S$ be the set of absolute values corresponding to a prime that divides a basis together with the usual Archimedian absolute value. For every non-Archimedian absolute values $\nu$ we choose $L_{\nu, i}\left(x_{1}, \ldots, x_{s}\right)=x_{i}$. The linear forms corresponding to the Archimedian absolute value are $L_{\infty, j}\left(x_{1}, \ldots, x_{s}\right)=x_{j}$ and $L_{\infty, i}\left(x_{1}, \ldots, x_{s}\right)=x_{j}-x_{i}$ for $i \neq j$. For $n$ large (such that $n^{2} \geq \max \left\{n+k_{i}+h_{i},\left(k_{i}+h_{i}\right)^{8 s / \varepsilon}\right\}$ for all $\left.i\right)$, we obtain the following inequality:

$$
\begin{aligned}
\overline{\mid \mathbf{x}}^{-\epsilon / 8} & =\left(\max _{i=1, \ldots, s}\left|n+k_{i}+h_{i}\right|\right)^{-\epsilon / 8} \\
& \geq n^{-\epsilon / 4} \geq n^{-\epsilon / 2} \max _{1 \leq i \leq s}\left(k_{i}+h_{i}\right)^{s} \\
& \geq \frac{C_{1} \cdots C_{s}}{\prod_{i \neq j} x_{i}} \prod_{i \neq j}\left|k_{j}+h_{j}-k_{i}-h_{i}\right| \\
& =\frac{x_{j}}{p_{j}^{\alpha_{j}}} \prod_{i \neq j} \frac{\left|k_{j}+h_{j}-k_{i}-h_{i}\right|}{p_{i}^{\alpha_{i}}} \\
& \geq \prod_{\nu \in S} \prod_{i=1}^{s}\left|L_{\nu, i}(\mathbf{x})\right|_{\nu} .
\end{aligned}
$$

The last inequality holds since $\left|L_{\infty, i}(\mathbf{x})\right|=\left|x_{j}-x_{i}\right|=\left|k_{j}+h_{j}-k_{i}-h_{i}\right|$ in case of $j \neq i$ and $\left|L_{\infty, j}(\mathbf{x})\right|=\left|x_{j}\right|$. Furthermore $\left|L_{p, i}(\mathbf{x})\right|_{p}=\left|C_{i} p_{i}^{\alpha_{i}}\right|_{p}=p^{-\alpha_{i}}$, if $p \mid p_{i}$ and $\left|L_{p, i}(\mathbf{x})\right|_{p} \leq 1$ otherwise. 
By Theorem 2 the solutions of this inequality lie only in finitely many subspaces. Therefore the vector $\mathbf{x}$ satisfies the linear system (let $T$ be an adequate subspaces):

$$
\begin{aligned}
x_{1} t_{1}+\cdots+x_{s} t_{s} & =0 & (\text { subspace } T), \\
x_{j}-x_{i} & =k_{j}+h_{j}-k_{i}-h_{i} & (i \neq j) .
\end{aligned}
$$

System (5) may be written in the following form

$$
\left(\begin{array}{cccccccc}
t_{1} & t_{2} & \cdots & t_{j-1} & t_{j} & t_{j+1} & \cdots & t_{s} \\
-1 & 0 & \cdots & 0 & 1 & 0 & \cdots & 0 \\
0 & -1 & \cdots & 0 & 1 & 0 & \cdots & 0 \\
\vdots & \vdots & \ddots & \vdots & \vdots & \vdots & \ddots & \vdots \\
0 & 0 & \cdots & -1 & 1 & 0 & \cdots & 0 \\
0 & 0 & \cdots & 0 & 1 & -1 & \cdots & 0 \\
\vdots & \vdots & \ddots & \vdots & \vdots & \vdots & \ddots & \vdots \\
0 & 0 & \cdots & 0 & 1 & 0 & \cdots & -1
\end{array}\right)\left(\begin{array}{c}
x_{1} \\
x_{2} \\
x_{3} \\
\vdots \\
x_{j} \\
x_{j+1} \\
\vdots \\
x_{s}
\end{array}\right)=\left(\begin{array}{c}
0 \\
k_{j}+h_{j}-k_{1}-h_{1} \\
k_{j}+h_{j}-k_{2}-h_{2} \\
\vdots \\
k_{j}+h_{j}-k_{j-1}-h_{j-1} \\
k_{j}+h_{j}-k_{j+1}-h_{j+1} \\
\vdots \\
k_{j}+h_{j}-k_{s}-h_{s}
\end{array}\right)
$$

Note that the $\mathbf{k}$-start Halton sequence avoids the corner $\mathbf{h}$ if and only if system (5) has infinitely many solutions. But the system has infinitely many solutions if and only if

$$
\sum_{i=1}^{s} t_{i}=0 \quad \text { and } \sum_{i \neq j} t_{i}\left(k_{i}+h_{i}\right)=-t_{j}\left(k_{j}+h_{j}\right) .
$$

But (6) yields at most $L^{s-1}$ possibilities for $\left(k_{1}+h_{1}, \ldots, k_{s}+h_{s}\right)$ such that $\max _{1 \leq i \leq s}\left(k_{i}+h_{i}\right) \leq$ $L$. Moreover, the Subspace theorem yields only a finite number $t$ of subspaces. Thus, we have at most $t L^{s-1}$ different vectors $\mathbf{k}$ that do not admit corner avoidance. Hence, $K(L) \leq t L^{s-1}$ and $\lim _{L \rightarrow \infty} \frac{\# K(L)}{L^{s}}=0$.

\section{Metric Results}

This section aims to provide metric results on corner properties of random start Halton sequences. It is shown that (Lebesgue) almost all z-start Halton sequences avoid corners. In the spirit of Lemma 1 we use the following notion to characterize points that are near the corners.

Definition 1. Let $f: \mathbb{Z}^{+} \rightarrow \mathbb{R}^{+}$be a fixed arithmetic function such that $f(n) \geq 1$ and

$$
\sum_{n=1}^{\infty} \frac{(\log (n f(n)))^{s-1}}{n f(n)}<\infty
$$

For $\mathbf{z}=\left(z_{1}, \ldots, z_{s}\right) \in \mathbb{Z}_{p_{1}} \times \cdots \times \mathbb{Z}_{p_{s}}$ and $n \geq 1$, let $\mathbf{x}_{n}=\left(\phi_{p_{1}}\left(n ; z_{1}\right), \ldots, \phi_{p_{s}}\left(n ; z_{s}\right)\right)$ be the $\mathbf{z}$-start Halton sequence in relatively coprime basis $p_{1}, \ldots, p_{s}$. The point $\mathbf{x}_{n}$ is said to be close to the corner $\mathbf{h}$ if there exist $\alpha_{1}, \ldots, \alpha_{s}$ such that for $i=1, \ldots, s$ :

$$
p_{i}^{\alpha_{i}} \|\left(z_{i}+h_{i}+n\right) \quad \text { and } \quad \prod_{i=1}^{s} p_{i}^{\alpha_{i}}>n f(n) .
$$

If (7) is fulfilled for infinitely many $n$, the $\mathbf{z}$-start Halton sequence $\left(\mathbf{x}_{n}\right)_{n \geq 1}$ is said to approach $\mathbf{h}$.

For the rest of this section we fix an arithmetic function $f$, which is assumed to fulfill the properties of Definition 1. 
Theorem 5. For $\mathbf{z}=\left(z_{1}, \ldots, z_{s}\right) \in[0,1)^{s}$ and $n \geq 1$, let

$$
\left(\mathbf{x}_{n}\right)_{n \geq 1}=\left(\mathbf{x}_{n}^{\mathbf{z}}\right)_{n \geq 1}=\left(\phi_{p_{1}}\left(n ; \tilde{\Phi}\left(z_{1}\right)\right), \ldots, \phi_{p_{s}}\left(n ; \tilde{\Phi}\left(z_{s}\right)\right)\right)_{n \geq 1}
$$

be the $\mathbf{z}$-start Halton sequence in relatively coprime basis $p_{1}, \ldots, p_{s}$. Then the set

$$
\mathcal{A}=\left\{\mathbf{z} \in[0,1)^{s}:\left(\mathbf{x}_{n}^{\mathbf{z}}\right)_{n \geq 1} \text { approaches a corner } \mathbf{h}\right\}
$$

has Lebesgue measure zero, i.e. $\lambda(\mathcal{A})=0$.

Proof. The proof will be done in several steps:

- A tiling for $\mathbb{Z}_{p_{1}} \times \cdots \times \mathbb{Z}_{p_{s}}$.

For $l_{1}, \ldots, l_{s} \in \mathbb{Z}^{+}$, let us define the fundamental tile by

$$
X_{l_{1}, \ldots, l_{s}}:=\left\{\mathbf{y} \in \mathbb{Z}_{p_{1}} \times \cdots \times \mathbb{Z}_{p_{s}}: p^{\alpha_{i}} \mid y_{i}, i=1, \ldots, s\right\} .
$$

A tiling of $\mathbb{Z}_{p_{1}} \times \cdots \times \mathbb{Z}_{p_{s}}$ is obtained by the tiles

$$
X_{l_{1}, \ldots, l_{s}}+\left(k_{1}, \ldots, k_{s}\right):=\left\{\mathbf{y} \in \mathbb{Z}_{p_{1}} \times \cdots \times \mathbb{Z}_{p_{s}}: p^{\alpha_{i}} \mid\left(y_{i}-k_{i}\right), i=1, \ldots, s\right\},
$$

for all integers $k_{i}$ with at most $\alpha_{i} p_{i}$-adic digits, i.e. $k_{i}<p_{i}^{l_{i}} \in \mathbb{Z}^{+},(i=1, \ldots, s)$.

Since $\Phi_{p}$ is surjective we also have a tiling of $[0,1]^{s}$. In most cases we will need a tiling induced by the fundamental tile $X_{l}:=X_{l, \ldots, l}$, for a fixed $l \in \mathbb{Z}^{+}$.

Remark: The tiles $X_{l_{1}, \ldots, l_{s}}+\left(k_{1}, \ldots, k_{s}\right)$ generate the $\sigma$-algebra of the Borel sets of the topological group $\mathbb{Z}_{p_{1}} \times \cdots \times \mathbb{Z}_{p_{s}}$. The Haar measure $\mu$ such that $\mu\left(\mathbb{Z}_{p_{1}} \times \cdots \times \mathbb{Z}_{p_{s}}\right)=1$ is obtained if we allocate to each tile $X_{l_{1}, \ldots, l_{s}}+\left(k_{1}, \ldots, k_{s}\right)$ the measure $p_{1}^{-l_{1}} \cdots p_{s}^{-l_{s}}$ (see [5, pages 202-3]). Moreover, the functions $\Phi$ and $\tilde{\Phi}$ are measurable and the Haar measure $\mu$ is induced by the Lebesgue measure $\lambda$ and vice versa, i.e. $\mu(A)=\lambda\left(\tilde{\Phi}^{-1}(A)\right)$ and $\lambda(B)=\mu\left(\Phi^{-1}(B)\right)$ for all measurable (Borel) sets $A \subseteq \mathbb{Z}_{p_{1}} \times \cdots \times \mathbb{Z}_{p_{s}}$ and $B \subseteq[0,1)^{s}$. To prove that $\Phi$ is measurable we only need to show that every element of a generating system of the Borel sets of $[0,1)^{s}$ has as preimage a Borel set of $\mathbb{Z}_{p_{1}} \times \cdots \times \mathbb{Z}_{p_{s}}$. In fact, we have

$$
\Phi^{-1}\left(\left[\frac{k_{1}}{p_{1}^{l_{1}}}, \frac{k_{1}+1}{p_{1}^{l_{1}}}\right) \times \cdots \times\left[\frac{k_{s}}{p_{s}^{l_{s}}}, \frac{k_{s}+1}{p_{s}^{l_{s}}}\right)\right)=X_{l_{1}, \ldots, l_{s}}^{-}+\left(k_{1}, \ldots, k_{s}\right),
$$

where $X_{l_{1}, \ldots, l_{s}}^{-}$is $X_{l_{1}, \ldots, l_{s}}$ without elements which have a component $y_{i} \in \mathbb{Z}_{p_{i}}$ of the form $y_{i}=\sum_{r=l_{i}+1}^{\infty}\left(p_{i}-1\right) p_{i}^{r}$. Loosely speaking $X_{l_{1}, \ldots, l_{s}}^{-}$is $X_{l_{1}, \ldots, l_{s}}$ without right upper border. It is easily seen that this right upper border is a Borel set, which shows that $\Phi$ is measurable, since also $X_{l_{1}, \ldots, l_{s}}$ is a Borel set.

Similarly we have

$$
\tilde{\Phi}^{-1}\left(X_{l_{1}, \ldots, l_{s}}+\left(k_{1}, \ldots, k_{s}\right)\right)=\left[\frac{k_{1}}{p_{1}^{l_{1}}}, \frac{k_{1}+1}{p_{1}^{l_{1}}}\right] \times \cdots \times\left[\frac{k_{s}}{p_{s}^{l_{s}}}, \frac{k_{s}+1}{p_{s}^{l_{s}}}\right] .
$$

Since the right side is a Borel set, $\tilde{\Phi}$ is measurable.

- Let $N \in \mathbb{Z}^{+}$be fixed, $l \in \mathbb{Z}^{+}$be large. Consider the tiling induced by $X_{l}$, if $\mathbf{x}_{N}^{\mathbf{z}}$ is close to the corner $\mathbf{0}$, then also the whole tile corresponding to $\mathbf{z}$ is close to the corner $\mathbf{0}$.

Define $\Phi(\mathbf{z}):=\left(\Phi_{p_{1}}\left(z_{1}\right), \ldots \Phi_{p_{s}}\left(z_{s}\right)\right)$. First, observe that for $i=1, \ldots, s$ and $a_{i} \in \mathbb{Z}_{p_{i}}$, we have

$$
\left(p_{i}^{\alpha_{i}} \mid\left(\tilde{\Phi}_{p_{i}}\left(z_{i}\right)+N\right)\right) \Longrightarrow\left(p_{i}^{\alpha_{i}} \mid\left(\tilde{\Phi}_{p_{i}}\left(z_{i}\right)+a_{i} p_{i}^{\alpha_{i}}+N\right)\right)
$$


For $i=1, \ldots, s$, let $\bar{k}_{i}=\sum_{r=0}^{\alpha_{i}} a_{r+1}\left(z_{i}\right) p_{i}^{r}$. From (8), we conclude that if $\mathbf{x}_{N}^{\mathbf{z}}$ is close to $\mathbf{0}$, also $\mathbf{x}_{N}^{\Phi(\overline{\mathbf{k}})}$ is close to $\mathbf{0}$. Thus, all points of the form $\mathbf{x}_{N}^{\Phi(\overline{\mathbf{k}}+\mathbf{y})}$ with $y \in X_{\alpha_{1}, \ldots, \alpha_{s}}$ are close to $\mathbf{0}$.

The same holds for all points of the form $\mathbf{x}_{N}^{\Phi(\overline{\mathbf{k}}+\mathbf{y})}$ with $y \in X_{\beta_{1}, \ldots, \beta_{s}}$, where $\beta_{i} \geq \alpha_{i}$ $(i=1, \ldots, s)$, in particular for $y \in X_{l}$ with $l \geq \max \left\{\alpha_{1}, \ldots, \alpha_{s}\right\}$.

- Reduction of the problem to a counting problem.

Let us define the set

$$
\mathcal{A}_{N}:=\left\{\mathbf{z} \in[0,1)^{s}: \mathbf{x}_{N}^{\mathbf{z}} \text { is close to } \mathbf{0}\right\} .
$$

The idea is to find a Borel measureable set $\mathcal{A}_{N}^{\prime} \supset \mathcal{A}_{N}$, such that we are able to estimate the measure of $\mathcal{A}_{N}^{\prime}$ by solving a counting problem. We denote the set of $s$-tuples of integers that possess $p_{i}$-adic expansions with at most $l$ digits by $\mathcal{F}_{(l)}$, i.e.

$$
\mathcal{F}_{(l)}=\mathbb{Z}^{s} \cap\left(\left[0, p_{1}^{l}\right) \times \cdots \times\left[0, p_{s}^{l}\right)\right)
$$

and consider the set

$$
\mathcal{C}_{N, l}=\left\{\left(k_{1}, \ldots, k_{s}\right) \in \mathcal{F}_{(l)}: \mathbf{x}_{N}^{\Phi\left(k_{1}, \ldots, k_{s}\right)} \text { is close to } \mathbf{0}\right\} .
$$

Let $l>\frac{\log (N f(N))}{\log 2}$, thus $l \geq \max \left\{\alpha_{1}, \ldots, \alpha_{s}\right\}$. We know that with $\mathbf{k} \in \mathcal{C}_{N, l}$ all $\mathbf{x}_{N}^{\Phi(\mathbf{k}+y)}$ are close to $\mathbf{0}$ provided $y \in X_{l}$. Therefore we define

$$
\mathcal{A}_{N}^{\prime}=\bigcap_{l=\left\lfloor\frac{\log (N f(N))}{\log 2}\right\rfloor+1}^{\infty} \mathcal{A}_{N, l}^{\prime} \quad \text { and } \quad \mathcal{A}_{N, l}^{\prime}=\Phi\left(\bigcup_{\mathbf{k} \in \mathcal{C}_{N, l}} \mathbf{k}+X_{l}\right) .
$$

Since the sets $\Phi\left(\mathbf{k}+X_{l}\right)$ are disjunct intervals with side lengths $p_{i}^{-l}$ we have

$$
\lambda\left(\mathcal{A}_{N, l}^{\prime}\right)=\frac{\sharp \mathcal{C}_{N, l}}{\left(p_{1} \cdots p_{s}\right)^{l}} .
$$

- Reduction to compute a volume.

We define the set $\mathcal{H}_{N, l}=\left\{\mathbf{x}_{N}^{\Phi(\mathbf{k})}: \mathbf{k} \in \mathcal{F}_{(l)}\right\}$ and the following tiling of $[0,1)^{s}$

$$
T_{\mathbf{k}, l}=\left[\frac{k_{1}}{p_{1}^{l}}, \frac{k_{1}+1}{p_{1}^{l}}\right) \times \cdots \times\left[\frac{k_{s}}{p_{s}^{l}}, \frac{k_{s}+1}{p_{s}^{l}}\right), \quad \mathbf{k} \in \mathcal{F}_{(l)} .
$$

We know that distinct elements of $\mathcal{H}_{N, l}$ lie in distinct sets $T_{\mathbf{k}, l}$. Suppose $\mathbf{u}, \mathbf{v} \in \mathcal{F}_{(l)}$ and $\mathbf{x}_{N}^{\Phi(\mathbf{u})}, \mathbf{x}_{N}^{\Phi(\mathbf{v})} \in T_{\mathbf{k}, l}$. Then for $i=1, \ldots, s, p_{i}^{l} \mid\left(\left(u_{i}+N\right)-\left(v_{i}+N\right)\right)$, thus $u_{i}-v_{i} \geq p_{i}^{l}$ or $u_{i}-v_{i}=0$. By $0 \leq u_{i}, v_{i}<p_{i}^{l}$, we have $\mathbf{u}=\mathbf{v}$.

In order to compute $\sharp \mathcal{C}_{N, l}$ we have to compute the number of elements of $\mathcal{H}_{N, l}$, which have hyperbolic distance less than $\frac{1}{N f(N)}$. As each element of $\mathcal{H}_{N, l}$ lies in one and only one tile $T_{\mathbf{k}, l}$, we get an upper bound on $\sharp \mathcal{C}_{N, l}$ by computing the number of tiles $T_{\mathbf{k}, l}$ possessing a left lower corner with hyperbolic distance less than $\frac{1}{N f(N)}$, i.e.

$$
\sharp \mathcal{C}_{N, l} \leq \sharp\left\{\mathbf{k} \in \mathcal{F}_{(l)}:\|\Phi(\mathbf{k})\|_{\mathbf{0}} \leq \frac{1}{N f(N)}\right\} .
$$

Thus, we have reduced the problem to count lattice points contained in some body. The number of lattice points contained in a body is about the volume of the body divided by the volume of the fundamental parallelotope plus the number of lattice points that lie near the border. Here, to each lattice point there is a parallelotope with side lengths $p_{i}^{-l},(i=1, \ldots, s)$ 
attached. Let $R$ denote the number of points lying on one of the hyper-planes characterized by $\left\{\mathbf{z} \in[0,1]^{s}: x_{i}=0\right\}$ for some $i=1, \ldots, s$. Furthermore define the sets

and

$$
\mathcal{C}_{N f(N)}=\left\{\mathbf{z} \in[0,1)^{s}: z_{1} \cdots z_{s}<\frac{1}{N f(N)}\right\}
$$

$$
\delta \mathcal{C}_{N f(N)}=\left\{\mathbf{z} \in[0,1)^{s}: z_{1} \cdots z_{s}=\frac{1}{N f(N)}\right\} .
$$

Set $\varepsilon:=\sqrt{p_{1}^{-2 l}+\cdots+p_{s}^{-2 l}}$. The area near the border is given by

$$
\Delta \mathcal{C}_{N f(N)}=\left\{\mathbf{z} \in\left(\mathbb{R}^{+}\right)^{s}:\left|\delta \mathcal{C}_{N f(N)}-\mathbf{z}\right|<\varepsilon\right\} \backslash \mathcal{C}_{N f(N)},
$$

where $\left|\delta \mathcal{C}_{N f(N)}-\mathbf{z}\right|$ denotes the minimal Euclidean distance from $\delta \mathcal{C}_{N f(N)}$ to $\mathbf{z}$.

Thus, a bound for $\sharp \mathcal{C}_{N, l}$ is given by

$$
\sharp \mathcal{C}_{N, l} \leq\left(\lambda\left(\mathcal{C}_{N f(N)}\right)+\lambda\left(\Delta \mathcal{C}_{N f(N)}\right)\right)\left(p_{1} \cdots p_{s}\right)^{l}+R,
$$

where $R$ is the number of lattice points lying in $\mathcal{C}_{N f(N)} \cap\left\{\mathbf{z} \in[0,1)^{s}: z_{i}=1\right\}$ for some $i \in\{1, \ldots, s\}$, i.e. the number of lattice points which lie on the remaining borders. By the definition of $R$ we find

$$
R \leq\left(p_{1} \cdots p_{s}\right)^{l} \sum_{j=1}^{s} p_{j}^{-l} \leq\left(p_{1} \cdots p_{s}\right)^{l} \sqrt{s} \varepsilon .
$$

Since $\delta \mathcal{C}_{N f(N)}$ is convex, we may project it on each hyper-plane $x_{i}=0$ to get a bound for the volume of $\Delta C_{N f(N)}$ of the form $s \epsilon$. This yields

$$
\sharp \mathcal{C}_{N, l} \leq\left(\lambda\left(\mathcal{C}_{N f(N)}\right)+(s+\sqrt{s}) \varepsilon\right)\left(p_{1} \cdots p_{s}\right)^{l} .
$$

- Computation of the volume

We claim:

$$
\lambda\left(\mathcal{C}_{k}\right)=\frac{1}{k} \sum_{j=0}^{s-1} \frac{(\log k)^{j}}{j !}<\frac{(\log k)^{s-1}}{k} e .
$$

Let us denote by $C_{k}^{(j)}$ the cylinder $\left\{\left(z_{1}, \ldots z_{s}\right) \in[0,1)^{s}: z_{1} \cdots z_{j} \leq 1 / k\right\}$. Once we have proved $\lambda\left(C_{k}^{(j+1)} \backslash C_{k}^{(j)}\right)=\frac{(\log k)^{j}}{k(j !)}$, the claim follows by induction.

$$
\begin{aligned}
\lambda\left(C_{k}^{(j+1)} \backslash C_{k}^{(j)}\right) & =\int_{z_{1}=\frac{1}{k}}^{1} \int_{z_{2}=\frac{1}{k z_{1}}}^{1} \cdots \int_{z_{j}=\frac{1}{k z_{1} \cdots z_{j-1}}}^{1} \int_{z_{j+1}=0}^{\frac{1}{k z_{1} \cdots z_{j}}} \int_{z_{j+2}=0}^{1} \cdots \int_{z_{s}=0}^{1} 1 d z_{s} \cdots d z_{1} \\
& =\int_{z_{1}=\frac{1}{k}}^{1} \int_{z_{2}=\frac{1}{k z_{1}}}^{1} \cdots \int_{z_{j}=\frac{1}{k z_{1} \cdots z_{j-1}}}^{1} \frac{1}{k z_{1} \cdots z_{j}} d z_{j} \cdots z_{1} \\
& =\int_{z_{1}=\frac{1}{k}}^{1} \int_{z_{2}=\frac{1}{k z_{1}}}^{1} \cdots \int_{z_{j-1}=\frac{1}{k z_{1} \cdots z_{j-2}}}^{1} \frac{\log \left(k z_{1} \cdots z_{j-1}\right)}{k z_{1} \cdots z_{j-1}} d z_{j-1} \cdots d z_{1} \\
& =\int_{z_{1}=\frac{1}{k}}^{1} \int_{z_{2}=\frac{1}{k z_{1}}}^{1} \cdots \int_{z_{j-2}=\frac{1}{k z_{1} \cdots z_{j-3}}}^{1} \frac{\left(\log \left(k z_{1} \cdots z_{j-2}\right)\right)^{2}}{2 k z_{1} \cdots z_{j-2}} d z_{j-2} \cdots d z_{1} \\
& =\cdots=\frac{(\log k)^{j}}{k(j !)} .
\end{aligned}
$$


- The probability that the $N$-th point of a Halton sequence is close to $\mathbf{0}$

Remember, that $\mathcal{A}_{N}^{\prime}:=\bigcap_{l=l_{0}}^{\infty} \mathcal{A}_{N, l}^{\prime}$, where $l_{0}=\left\lfloor\frac{\log (N f(N))}{\log 2}\right\rfloor+1$. Since $\mathcal{A}_{N, l+1}^{\prime} \subset \mathcal{A}_{N, l}^{\prime}$, the set $A_{N}^{\prime}$ is measurable.

The volume of $A_{N, l}^{\prime}$ may now be estimated by

$$
\lambda\left(\mathcal{A}_{N, l}^{\prime}\right) \leq e \frac{(\log (N f(N)))^{s-1}}{N f(N)}+(s+\sqrt{s}) \varepsilon .
$$

Since $\varepsilon \rightarrow 0$ as $l \rightarrow \infty$, we conclude

$$
\lambda\left(\mathcal{A}_{N}^{\prime}\right) \leq e \frac{(\log (N f(N)))^{s-1}}{N f(N)} .
$$

- The probability that the random start Halton sequence is approaching $\mathbf{0}$

Let us define the sets

$$
\begin{aligned}
\mathcal{B}_{0} & :=\left\{\mathbf{z}:\left(\mathbf{x}_{n}^{\mathbf{z}}\right)_{n \geq 1} \text { is approaching } \mathbf{0}\right\} \text { and } \\
\mathcal{B}_{0}^{(k)} & :=\left\{\mathbf{z}:\left(\mathbf{x}_{n}^{\mathbf{z}}\right)_{n \geq 1} \text { has at least } k \text { points that are close to } \mathbf{0}\right\} .
\end{aligned}
$$

Hence, $\mathcal{B}_{0}^{(k+1)} \subset \mathcal{B}_{0}^{(k)}$ and

$$
\bigcap_{k=1}^{\infty} \mathcal{B}_{0}^{(k)}=\mathcal{B}_{0}
$$

If in a sequence $\left(\mathbf{x}_{n}\right)$ there are $k$ points close to $\mathbf{0}$, then the index $N_{k}$ of the $k$-th point $\mathbf{x}_{N_{k}}$ that is close to $\mathbf{0}$ is in the set $\left\{N \in \mathbb{Z}^{+}: N \geq k\right\}$. Therefore

$$
\mathcal{B}_{0}^{(k)} \subset \bigcup_{N=k}^{\infty} \mathcal{A}_{N}^{\prime} \text {. }
$$

If we take into account the definition of $\mathcal{B}_{0}$, then

$$
\mathcal{B}_{0} \subset \mathcal{B}_{0}^{\prime}:=\bigcap_{k=1}^{\infty} \bigcup_{N=k}^{\infty} \mathcal{A}_{N}^{\prime} .
$$

Since the Lebesgue measure is continuous, we find

$$
\mathcal{B}_{0}^{\prime}=\lim _{k \rightarrow \infty} \sum_{N=k}^{\infty} \lambda\left(\mathcal{A}_{N}^{\prime}\right) \leq \lim _{k \rightarrow \infty} \sum_{N=k}^{\infty} e \frac{(\log (N f(N)))^{s-1}}{N f(N)}=0,
$$

by the assumption $\sum_{N=1}^{\infty} \frac{\left(\log (N f(N))^{s-1}\right.}{N f(N)}<\infty$. Finally, $\lambda\left(\mathcal{B}_{0}^{\prime}\right)=0$ includes $\lambda\left(\mathcal{B}_{0}\right)=0$ by the completeness of the Lebesgue measure.

- Deduction from one corner to all corners.

If a sequence $\left(\mathbf{x}_{n}^{\mathbf{z}}\right)_{n \geq 1}$ approaches a corner $\mathbf{h} \in\{0,1\}^{s}$, then the sequence $\left(\mathbf{x}_{n}^{\mathbf{z}^{\prime}}\right)_{n \geq 1}$ with $z_{i}^{\prime}=\Phi_{p_{i}}\left(\tilde{\Phi}_{p_{i}}\left(x_{i}\right)-h_{i}\right)$ approaches the corner $\mathbf{0}$. Thus, the probability for a random start Halton sequence to approach any corner is $2^{s}$ times the probability for approaching $\mathbf{0}$. Hence, $\lambda(\mathcal{A})=0$.

Finally, let us give the following corollary. 
Corollary 1. Almost all random-start Halton sequences avoid all corners, i.e. for any fixed $\epsilon>0$ there exists a constant $c=c\left(\epsilon, p_{1}, \ldots, p_{s}\right)>0$ such that for any corner $\mathbf{h} \in\{0,1\}^{s}$, and all $n \geq 1$ the condition

is fulfilled.

$$
\left\|\mathbf{x}_{n}\right\|_{\mathbf{h}}>c n^{-1-\epsilon}
$$

Proof. The result is a direct consequence of Theorem 5 and Lemma 1 combined with the fact that

$$
\sum_{n=1}^{\infty} \frac{\log \left(n^{1+\epsilon}\right)^{s-1}}{n^{1+\epsilon}}=\sum_{n=1}^{\infty} \frac{(1+\epsilon)^{s-1} \log (n)^{s-1}}{n^{1+\epsilon}}<\infty .
$$

Remark: Theorem 4 could also be proved by similar methods (counting lattice points in hyperbolic areas) as used in the proof of Theorem 5. Although we gave a "Diophantine Proof" of Theorem 4, because it is completely different and moreover it provides more information on the structure, which points avoid corners. For example it is possible to show that the (non-randomized) Halton sequence avoids all corners with the "Diophantine approach" (cf. [4]).

On the other hand we could also use Theorem 4 in the proof of Theorem 5. Indeed instead of counting lattice points one could apply Theorem 4 directly to estimate the number of starting points that come close to a corner. But in this case we had to restrict the proof to the case $f(n)=n^{\epsilon}$.

At last note that in Corollary 1 the factor $\frac{c}{n^{1+\epsilon}}$ could be replaced by $\frac{c}{n(\log n)^{s} \log _{2} n \cdots \log _{l-1} n\left(\log _{l} n\right)^{1+\epsilon}}$, where $\log _{k} n$ is the $k$ times iterated logarithm of $n$.

\section{REFERENCES}

[1] H. P. Evertse, Jan-Hendrik Schlickewei. A quantitative version of the Absolute Subspace Theorem. J. Reine Angew. Math., 548:21-127, 2002.

[2] H. Faure. Discrépance de suites associées à un système de numération (en dimension un). Bull. Soc. Math. France, 109:143-182, 1981

[3] J. H. Halton. On the efficiency of certain quasi-random sequences of points in evaluating multidimensional integrals. Numer. Math., 2:84-90, 1960 .

[4] J. Hartinger, R. F. Kainhofer, and V. Ziegler. On the corner avoidance properties of various low-discrepancy sequences. INTEGERS- Electronic Journal of Combinatorial Number Theory, (5(3)), 2005.

[5] E. Hewitt and K. A. Ross. Abstract Harmonic Analysis I, volume 115 of Grundlehren der Mathematischen Wisssenschaften. Springer-Verlag, Berlin, 1963.

[6] E. Hlawka. Uniform distribution modulo 1 and numerical analysis. Compositio Math., 16:92-105, 1964.

[7] H. Niederreiter. Point sets and sequences with small discrepancy. Monatsh. Math., 104:273-337, 1987.

[8] H. Niederreiter. Low-discrepancy and low-dispersion sequences. Journal of Number Theory, 30(1):51-70, 1988.

[9] H. Niederreiter. Random number generation and Quasi-Monte Carlo methods, volume 63 of SIAM Conf. Ser. Appl. Math. SIAM, Philadelphia, 1992.

[10] A. B. Owen. Randomized qme and point singularities. Technical Report, Stanford University, 2004.

[11] A. B. Owen. Multidimensional variation for Quasi-Monte Carlo. In J. Fan and G. Li, editors, International Conference on Statistics in Honour of Professor Kai-Tai Fang's 65th Birthday, 2005.

[12] A. B. Owen. Halton sequences avoid the origin. SIAM Review, 48, 2006, (to appear).

[13] H. P. Schlickewei. Die p-adische Verallgemeinerung des Satzes von Thue-Siegel-Roth-Schmidt. J. Reine Angew. Math., 288:86-105, 1976.

[14] W. M. Schmidt. Diophantine approximations and Diophantine equations, volume 1467 of Lecture Notes in Mathematics. Springer-Verlag, Berlin, 1991

[15] I. M. Sobol'. On the distribution of points in a cube and the approximate evaluation of integrals. USSR Comput. Math. Math. Phys., 7:86-112, 1967.

[16] I. M. Sobol'. On the use of uniformly distributed sequences for approximate computations of improper integrals. In S. Sobolev, editor, Theory of cubature formulas and applications to certain problems in mathematical physics, pages 62-66. Novosibirsk Nauka, 1973. 
[17] S. Tezuka and T. Tokuyama. A note on polynomial arithmetic analogue of halton sequences. ACM Trans. Model. Comput. Simul., 4(3):279-284, 1994.

[18] X. Wang and F. J. Hickernell. Randomized Halton sequences. Math. Comput. Modelling, 32(7-8):887-899, 2000.

Johann Radon Institute for Computational and Applied Mathematics (RICAM), Austrian Academy of Sciences, Altenbergerstrasse 69, A-4040 Linz, Austria

E-mail address: juergen.hartinger@oeaw.ac.at

Institut für Mathematik, University of Natural Resources and Applied Life Sciences, Gregor-MendelStrasse 33, A-1180 Vienna, Austria

E-mail address: ziegler@finanz.math.tugraz.at 